Communications in Physics, Vol. 29, No. 2 (2019), pp. 119-128

DOI:10.15625/0868-3166/29/2/13508

\title{
MAGNETIC ORDER IN HEISENBERG MODELS ON NON-BRAVAIS LATTICE: POPOV-FEDOTOV FUNCTIONAL METHOD
}

\author{
PHAM THI THANH NGA ${ }^{1, \dagger}$ AND NGUYEN TOAN THANG ${ }^{2}$ \\ ${ }^{1}$ Thuy loi University, 175 Tay Son, Dong Da dist., Hanoi, Vietnam \\ ${ }^{2}$ Institute of Physics, Vietnam Academy of Science and Technology, \\ 10 Dao Tan, Ba Dinh dist., Hanoi, Vietnam \\ ${ }^{\dagger} E$-mail: nga_ptt@tlu.edu.vn
}

Received 27 December 2018

Accepted for publication 13 March 2019

Published 10 May 2019

\begin{abstract}
We study magnetic properties of ordered phases in the Heisenberg model on a nonBravais lattice by means of a Popov - Fedotov trick, which takes into account a rigorous constraint of a single occupancy. We derive the magnetization and the free energy using sadle point approximation in the functional integral formalism. We illustrate the application of the Popov-Fedotov approach to the Heisenberg antiferromagnet on a honeycomb lattice.
\end{abstract}

Keywords: Popov - Fedotov trick, functional integral, Heisenberg model, non-Bravais lattice.

Classification numbers: 71.10.Fd, 71.27.ea, 71.10.-b, 75.10.Jm, 75.30.Ds.

\section{INTRODUCTION}

It is impossible to use the Wick's theorem for the spin operators because they are neither bosonic nor fermionic [1]. Therefore, the powerful methods of many body theory such as diagrammatic techniques and functional integral representations for spin systems are substantially more complicated than those for boson or fermion systems. Many versions of the functional integral formalism have been developed. Some of them are dealing directly with spin operators [2]. However, the corresponding rules for summation of series in high orders contain the combinatoric rules and in many cases are very complicated [2]. Another method is based on coherent states for spins which is applicable only at low temperatures (no-linear $\sigma$ model) [3]. Other techniques based on expressing the spin operators in terms of fermionic or bosonic operators [4] are faced to the problem of the local constraint. The representation of spins as a bilinear combination of auxiliary canonical operators increases the dimensionality of Hilbert space where these operators

(C)2019 Vietnam Academy of Science and Technology 
act. As a result, the unphysical states should be removed from the consideration by some local constraint conditions, where the number of auxiliary particles on each site is fixed. Due to the constraint requirement standard many-body methods cannot be applied. There are several ways of circumventing this difficulty. In the most simple approach the exclusion of the spurious unphysical states is cured by a replacement of the local constraint by a so called global constraint where the number of auxiliary particles is fixed merely on the thermal average. It may be done by introducing a Lagrange multiplier and the conventional many-body technique can be used. But such a replacement makes approximations for quantum spin systems to be uncontrolled.

In 1988, Popov and Fedotov proposed [5] a simple approach for quantum spin-1/2 and spin1 systems that is free of the local constraint. They found that the partition function for spin systems can be reformulated in terms of Fermi operators, where an imaginary chemical potential was introduced to eliminate statistical contributions from unphysical states. Latter the extension of the Popov-Fedotov method was derived for arbitrary spin [6,7]. Recently, the Popov-Fedotov trick has been successfully combined with the bold diagrammatic Monte-Carlo method to study frustrated quantum systems [8]. The Popov-Fedotov fermionization technique has been also generalized for strongly correlated systems $[9,10]$. For specific magnetic Heisenberg systems, the Popov-Fedotov approach has been applied to study magnetic properties of spin-1/2 systems on Bravais lattices such as ferromagnet [11], antiferromagnet on hypercubic and square lattices [12,13], antiferromagnet on triangular lattice [14]. The Popov-Fedotov method has been applied successfully also to the negative-U Hubbard model [15], spin glass model [16], Kondo lattice model [17]...

In this paper we apply the Popov-Fedotov procedure to the problem of ordered phases in Heisenberg models on non-Bravais lattices. It is motivated by the fact that magnets on nonBravais lattices have been extensively investigated from both the theoretical and experimental viewpoints in recent years because such systems display rich and interesting behaviors due to the strong interplay between quantum fluctuations and frustration [18]. New and fascinating phase structures have been studied for the Heisenberg model on an Union Jack lattice [19], a crossstriped square lattice [20], a planar pyrochlore lattice [21], a chevron-square lattice [22]. Particular interest has been focused on the honeycomb [23,24] and Kagome lattices [25], because the magnon dispersions in these lattices show similar features to topological insulators in electronic systems leading to topological magnon effect.

The paper is organized as follows. In Sec. II, we present general model on non-Bravais lattice and the Popov-Fedotov formalism. In Sec. III, we explicate details of the calculation procedure in mean-field and one-loop approximations for the case of a lattice with two sites per unit cell. In Sec. IV, the results for a particular model on the honeycomb lattice are derived. We end with a brief summary and discussions in Sec. V.

\section{THE FORMALISM}

We consider a Heisenberg model on a general non-Bravais lattice with $n$-sites per unit cell. The Hamiltonian of the system reads:

$$
H=\sum_{i j} J_{i j} \vec{S}_{i} \cdot \vec{S}_{j}
$$

We start by determining the classical ground state with assuming coplanar magnetic structure, which can be shown for the case of isotropic exchange interactions $J_{i j}$. In the classical limit 
the spins on site $i$ may be parameterized as:

$$
\vec{S}_{i}=S\left[\cos \left(\vec{Q} \vec{r}_{i}+\phi_{i}\right) \vec{u}+\sin \left(\vec{Q} \vec{r}_{i}+\phi_{i}\right) \vec{v}\right]
$$

with $\vec{u}$ and $\vec{v}$ being two orthogonal unit vectors in the spin plane. Vertor $\vec{Q}$ is the ordering vector and $\phi_{i}$ is the angle between $i$-spin vector and some fixed direction in the spin plane. The parameter $\vec{Q}$ and $\phi_{i}$ should be found by minimizing the classical energy, which in term of the ordering vector $\vec{Q}$ and the angle $\phi_{i}$ has the following form:

$$
E_{c l}=\frac{1}{2} S^{2} \sum_{i j} J_{i j} \cos \Delta_{i j}
$$

where

$$
\Delta_{i j}=\vec{Q} \vec{\delta}_{i j}+\left(\phi_{j}-\phi_{i}\right)
$$

and $\vec{\delta}_{i j}$ being a vector connecting a site $i$ with a site $j$. Note that the classical energy depends on the angle between the spins in the unit cell only through $\left(\phi_{j}-\phi_{i}\right)$ so one can choose one angle $\phi_{i}$ to be zero. As a result the classical state may be defined by $\mathrm{n}$ parameters. Depending on the exchange interaction and lattice structure there may exist different sets of parameters $\left\{\vec{Q}, \phi_{i}\right\}$ corresponding to different ordered phases.

When we represent the spin operators in terms of auxiliary fermion or boson ones we should choose some spin quantization axis, say Oz-axis. In order to take into account the fluctuation contribution it is convenient to choose the spin quantization axis along the classical spin orientation. In general, the spin direction may be different from site to site. Following Miyake [26], we transform the spin components $\left\{S_{i}^{x}, S_{i}^{y}, S_{i}^{z}\right\}$ from the laboratory reference frame to the local reference frame $\left\{S_{i}^{x^{\prime}}, S_{i}^{y^{\prime}}, S_{i}^{z^{\prime}}\right\}$ at each site in such a way that the spin quantization axis represents the local classical spin orientation:

$$
\left\{\begin{array}{c}
S_{i}^{z}=S_{i}^{z^{\prime}} \cos \theta_{i}-S_{i}^{x^{\prime}} \sin \theta_{i}, \\
S_{i}^{x}=S_{i}^{z^{\prime}} \sin \theta_{i}-S_{i}^{x^{\prime}} \cos \theta_{i}, \\
S_{i}^{y}=S_{i}^{y^{\prime}} .
\end{array}\right.
$$

Due to the transformation (5) in the following one needs to introduce only one kind of auxiliary fermions for all sites

Substituting (5) in (1), we get:

$$
H=-\frac{1}{2} \sum_{\substack{i, j \\ \alpha, \beta=x, y, z}} J_{i j}^{\alpha \beta} S_{i}^{\alpha} S_{j}^{\beta}
$$

The exchange couplings in the local reference frame have the following form:

$$
\left\{\begin{array}{l}
J_{i j}^{x x}=J_{i j}^{z z}=X_{i j}=-J_{i j} \cos \left(\Delta_{i j}\right), \\
J_{i j}^{y y}=Y_{i j}=-J_{i j}, \\
J_{i j}^{z x}=-J_{i j}^{z x}=W_{i j}=J_{i j} \sin \left(\Delta_{i j}\right), \\
J_{i j}^{x y}=J_{i j}^{y x}=J_{i j}^{y z}=J_{i j}^{z y}=0 .
\end{array}\right.
$$


According to Popov and Fedotov [5] we use the following the representation for the spin$1 / 2$ operator:

$$
S_{i}^{\alpha}=\frac{1}{2} \sum_{\sigma \sigma^{\prime}} a_{i \sigma}^{+} \sigma_{\sigma \sigma^{\prime}}^{\alpha} a_{i \sigma^{\prime}}
$$

where $\sigma=\left(\sigma^{x}, \sigma^{y}, \sigma^{z}\right)$ are the Pauli matrices, and $\sigma, \sigma^{\prime}=\uparrow, \downarrow$ are the spin indices. The Fock state of the fermion $a_{i \sigma}$ is spanned by four states. Among them the unphysical states $|0\rangle ;|2\rangle=a_{i \uparrow}^{+} a_{i \downarrow}^{+}|0\rangle$ where $|0\rangle$ is the vacuum should be excluded by the constraint at each site:

$$
\hat{N}_{i}=\sum_{\sigma} a_{i \sigma}+a_{i \sigma}=1
$$

The constraint may be enforced by introducing the projection operator $\hat{P}=\frac{1}{i^{N}} e^{i \frac{\pi}{2} \sum_{i} \hat{N}_{i}}$ to the partition function

$$
Z=\operatorname{Tr}\left[e^{-\beta \hat{H}} \hat{P}\right]
$$

with $\hat{H}$ being Hamiltonian (6), written in terms of the auxiliary operators (8). Because the trace over unphysical states at each site vanishes, the contributions of the unphysical states to the partition function cancel out one with others. Therefore, the partition function describing the Hamiltonian (6) with exactly one spin per site is given by

$$
Z=\left(\frac{1}{i^{N}}\right) \operatorname{Tr} e^{-\beta(\hat{H}-\mu \hat{N})},
$$

where $N$ denotes the site number and $\mu=\frac{i \pi}{2 \beta}$ is the purely imaginary Lagrange multiplier playing the role of imaginary chemical potential of the auxiliary fermion system. As a result, after performing Fourier transformation over imaginary time, the fermionic Matsubara frequences are modified to have the following form

$$
\tilde{\omega}_{F}=\omega_{F}-\frac{\pi}{2 \beta}=\frac{2 \pi}{\beta}\left(n+\frac{1}{4}\right) .
$$

The further calculation may be carried out following the main steps as in Ref. [14]. First we represent the partition function as a functional integral over the coherent state Grassmann variables. Then we perform a Hubbard-Stratonovich transformation introducing the Bose auxiliary vector field $\vec{\phi}_{i}(\Omega)$ to get rid of the 4-fermion terms. Next we integrate out the Grassmann variables to get the partition function in terms of the Bose auxiliary vector field $\vec{\phi}_{i}(\Omega)$ only. In order to apply a perturbation technique, we decompose the auxiliary Bose field as follows

$$
\vec{\phi}_{i}(\Omega)=\vec{\phi}_{i 0}(\Omega=0)+\delta \vec{\phi}_{i}(\Omega),
$$

where $\vec{\phi}_{i 0}(\Omega=0) \equiv \vec{\phi}_{i 0}$ is the mean field part defined from the least action principle and is related to the classical ground states magnetization per site $\vec{m}_{i 0}$ as follows

$$
\phi_{i 0}^{\alpha}=\sum_{j \beta} m_{j 0}^{\beta} J_{i j}^{\beta \alpha} .
$$


Because only the $z$-components of $\vec{m}_{i 0}$ and $\vec{\phi}_{i 0}$ are non-zero in the above chosen local reference frame, $m_{i 0}^{\alpha}=m_{i 0} \delta_{\alpha, z} ; \phi_{i 0}^{\alpha}=\phi_{i 0} \delta_{\alpha, z}$, then the mean-field equation of the magnetization reads

$$
m_{i 0}=\frac{1}{2} \tanh \frac{\beta}{2} \sum_{j} J_{i j}^{z z} m_{j 0} .
$$

Correspondingly, the mean-field free energy is given by

$$
F_{M F}=\frac{1}{2} \sum_{i j} J_{i j}^{z z} m_{i 0} m_{j 0}+\sum_{i} \ln \left(2 \cosh \left(\frac{1}{2} \beta \phi_{i 0}\right)\right) .
$$

To separate the transverse and longitudinal fluctuations we set $\delta \phi_{i}^{ \pm}(\Omega)=\delta \phi_{i}^{x}(\Omega) \pm i \delta \phi_{i}^{y}(\Omega)$. Then the fluctuation contribution in the one loop approximation to the free energy has the following form

$$
\delta F_{f l}=\frac{1}{2 \beta} \ln \operatorname{det} \hat{D}_{i j}(\Omega),
$$

where

$$
\hat{D}_{i j}(\Omega)=\hat{I}+\hat{J}_{i j} \hat{K}_{i j}(\Omega) .
$$

In the basics $(+,-, z)$ the elements of the coupling matrix $\hat{J}_{i j}$ are defined as follows

$$
\left\{\begin{array}{l}
J_{i j}^{++}=J_{i j}^{--}=X_{i j}-Y_{i j}, \\
J_{i j}^{+-}=J_{i j}^{-+}=X_{i j}+Y_{i j}, \\
J_{i j}^{z z}=X_{i j}, \\
J_{i j}^{+z}=J_{i j}^{-z}=-J_{i j}^{z+}=-J_{i j}^{z-}=-W_{i j} .
\end{array}\right.
$$

The none-zero elements of the matrix $\hat{K}_{i j}(\Omega)$ are given as

$$
\left\{\begin{array}{l}
K_{i j}^{+-}(\Omega)=\left(K_{i j}^{-+}(\Omega)\right)^{*}=\delta_{i j} k_{T}(\Omega) ; k_{T}(\Omega)=\frac{\beta}{2} \frac{m_{i 0}}{\phi_{i 0}+i \Omega} \\
K_{i j}^{z z}(\Omega)=\delta_{i j} \delta_{\Omega, 0} k_{z} ; k_{z}=m_{i 0}^{2}-\frac{1}{4} .
\end{array}\right.
$$

It is convenient to perform the Fourier transformation over the coordinates $\vec{r}_{i}$ and $\vec{r}_{j}$ of $\hat{D}_{i j}$ before calculating det $\hat{D}_{i j}$. In the case of a Bravais lattice all sites are equivalent so $\hat{D}(\vec{p})$ is a $3 \times 3$ matrix. In a non-Bravais lattice with $n$ site in a unit cell the matrix $\hat{D}(\vec{p})$ is $3 n \times 3 n$ block matrix. In this case $\operatorname{det} \hat{D}(\vec{p})$ be calculated following Silvester [27] and Powell [28], who show the determinant of a matrix with $k^{2}$ blocks can be reduced to the product of the determinants of $k$ distinct combinations of single block.

For example, for a matrix $\hat{M}$ having 4 blocks

$$
\operatorname{det} \hat{M}=\operatorname{det}\left(\begin{array}{cc}
\hat{A} & \hat{B} \\
\hat{C} & \hat{D}
\end{array}\right)=\operatorname{det}\left(\hat{A} \hat{D}-\hat{B} \hat{D}^{-1} \hat{C} \hat{D}\right),
$$

if $\hat{D}$ is invertibe. If different blocks of $\hat{M}$ commute, the Eq. (21) takes a simple form. For example, if $\hat{C} \hat{D}=\hat{D} \hat{C}$ then

$$
\operatorname{det} \hat{M}=\operatorname{det}(\hat{A} \hat{D}-\hat{B} \hat{C}) .
$$

In what follows in the next section we shall use the formula (21) for the case of non-Bravais lattice with two sites in a unit cell. 


\section{LATTICE WITH TWO SITES PER UNIT CELL}

Let $A$ and $B$ refer to the two lattice points in the unit cell. We can choose the angle $\phi_{A}=0$ and $\phi_{B}=\phi$. Hence the classical ground state is determined by two parameters $\vec{Q}$ and $\phi$. We define the Fourier transformation of the coupling $J_{i j}$ along the $i j$-bond

$$
J(\vec{p})=\frac{2}{N} \sum_{i j} J_{i j} e^{-i \vec{p}\left(\vec{r}_{i}-\vec{r}_{j}\right)} .
$$

Because the sites $i$ and $j$ may belong to $A$ or $B$ sublattice, then from (23) we have

$$
J_{\alpha \alpha^{\prime}}(\vec{p})=\sum_{\vec{\delta}_{\alpha \alpha^{\prime}}} J_{\alpha \alpha^{\prime}} e^{-i \vec{p} \vec{\delta}_{\alpha \alpha^{\prime}}},
$$

where $\alpha, \alpha^{\prime}=A, B$.

From (7) and (24) one derives

$$
\left\{\begin{aligned}
X(\vec{p}) & =-\frac{1}{2}(J(\vec{p}-\vec{Q})+J(\vec{p}+\vec{Q})), \\
X_{A B}(\vec{p}) & =X_{B A}^{*}(\vec{p})=-\frac{1}{2}\left(J_{A B}(\vec{p}-\vec{Q}) e^{i \phi}+J_{B A}(\vec{p}+\vec{Q}) e^{-i \phi}\right), \\
Y_{\alpha \alpha^{\prime}}(\vec{p}) & =-J_{\alpha \alpha^{\prime}}(\vec{p}), \\
W_{\alpha \alpha}(\vec{p}) & =-\frac{i}{2}\left(J_{\alpha \alpha}(\vec{p}-\vec{Q})-J_{\alpha \alpha}(\vec{p}+\vec{Q})\right), \\
W_{A B}(\vec{p}) & =-W_{B A}^{*}(\vec{p})=-\frac{i}{2}\left(J_{A B}(\vec{Q}-\vec{p}) e^{-i \phi}-J_{A B}(-\vec{Q}-\vec{p}) e^{i \phi}\right) .
\end{aligned}\right.
$$

The Fourier transformation of the matrix $\hat{D}_{i j}(\Omega)$ has the following block form

$$
\hat{D}(\vec{p}, \Omega)=\left(\begin{array}{cc}
\hat{D}_{A A}(\vec{p}, \Omega) & \hat{D}_{A B}(\vec{p}, \Omega) \\
\hat{D}_{B A}(\vec{p}, \Omega) & \hat{D}_{B B}(\vec{p}, \Omega)
\end{array}\right)
$$

Here the components $\hat{D}_{\alpha \alpha^{\prime}}(\vec{p}, \Omega)$ are $3 \times 3$ matrix given by

$$
\begin{gathered}
\hat{D}_{\alpha \alpha^{\prime}}(\vec{p}, \Omega)=I \delta_{\alpha \alpha^{\prime}}-R_{\alpha \alpha^{\prime}}(\vec{p}, \Omega) \\
R_{\alpha \alpha^{\prime}}=\left(\begin{array}{ccc}
\left(X_{\alpha \alpha^{\prime}}(\vec{p})+Y_{\alpha \alpha^{\prime}}(\vec{p})\right) k_{T}^{*}(\Omega) & \left(X_{\alpha \alpha^{\prime}}(\vec{p})-Y_{\alpha \alpha^{\prime}}(\vec{p})\right) k_{T}(\Omega) & -W_{\alpha \alpha^{\prime}}(\vec{p}) \delta_{\Omega, 0} k_{z} \\
\left(X_{\alpha \alpha^{\prime}}(\vec{p})-Y_{\alpha \alpha^{\prime}}(\vec{p})\right) k_{T}^{*}(\Omega) & \left(X_{\alpha \alpha^{\prime}}(\vec{p})+Y_{\alpha \alpha^{\prime}}(\vec{p})\right) k_{T}(\Omega) & -W_{\alpha \alpha^{\prime}}(\vec{p}) \delta_{\Omega, 0} k_{z} \\
W_{\alpha \alpha^{\prime}}(\vec{p}) k_{T}^{*}(\Omega) & W_{\alpha \alpha^{\prime}}(\vec{p}) k_{T}(\Omega) & X_{\alpha \alpha^{\prime}}(\vec{p}) \delta_{\Omega, 0} k_{z}
\end{array}\right) .
\end{gathered}
$$

Now we can use the formula (21) to calculate the $6 \times 6$ matrix $\hat{D}(\vec{p}, \Omega)(27)$. For simplicity we consider the case of nearest - neighbor bonding, which means $J_{\alpha \alpha}=0$. As a result the matrices $\hat{D}_{\alpha \alpha}(\vec{p}, \Omega)$ are $3 \times 3$ unit matrices. Then Eq. (21) hold and together with Eq. (25) and (28) leads to a simple expression for the determinant of the matrix $\hat{D}(\vec{p}, \Omega)$

$$
\operatorname{det} \hat{D}(\vec{p}, \Omega)=\operatorname{det}\left(\hat{I}+\hat{R}_{A B}(\vec{p}, \Omega) \hat{R}_{B A}(\vec{p}, \Omega)\right) .
$$

From (28) and (29) we derive

$$
\operatorname{det} \hat{D}(\vec{p}, \Omega)=\prod_{\vec{p}} \prod_{\Omega}\left(Q(\vec{p}, \Omega)+P(\vec{p}) \delta_{\Omega, 0}\right),
$$


where

$$
\begin{gathered}
Q(\vec{p}, \Omega)=1+4\left(X_{A B}^{*}(\vec{p}) Y_{A B}(\vec{p})+X_{A B}(\vec{p}) Y_{A B}^{*}(\vec{p})\right)\left|k_{T}(\Omega)\right|^{2} \\
+16\left|X_{A B}(\vec{p})\right|^{2}\left|Y_{A B}(\vec{p})\right|^{2}\left|k_{T}(\Omega)\right|^{4} \\
\quad-\left|X_{A B}(\vec{p})+Y_{A B}(\vec{p})\right|^{2}\left(k_{T}^{*}+k_{T}\right)^{2}, \\
P(\vec{p})=\left(1-4 k_{T}^{2}(0)\right)\left|Y_{A B}(\vec{p})\right| \\
\times\left[\begin{array}{l}
4 k_{T}^{2}(0) k_{z}^{2}\left(X_{A B}^{2}(\vec{p})+W_{A B}^{2}(\vec{p})\right)\left(X_{A B}^{* 2}(\vec{p})+W_{A B}^{* 2}(\vec{p})\right) \\
-\left|X_{A B}(\vec{p})\right|^{2} k_{z}^{2}-4\left|W_{A B}(\vec{p})\right|^{2} k_{T}(0) k_{z}
\end{array}\right] .
\end{gathered}
$$

Substituting (20) into (31), one rewrites (31) in the following form

$$
Q(\vec{p}, \Omega)=\frac{\left((i \Omega)^{2}-E_{1}^{2}(\vec{p})\right)\left((i \Omega)^{2}-E_{2}^{2}(\vec{p})\right)}{\left((i \Omega)^{2}-\phi_{0}^{2}\right)^{2}}
$$

where the magnon energies are given by

$$
\left\{\begin{aligned}
E_{1,2}(\vec{p})= & \phi_{0} \omega_{1,2}(\vec{p}) \\
\omega_{1,2}^{2}(\vec{p})= & +\frac{1}{2}\left(\frac{m_{0}}{\phi_{0}}\right)^{2}\left(X_{A B}^{*}(\vec{p}) Y_{A B}(\vec{p})+X_{A B}(\vec{p}) Y_{A B}^{*}(\vec{p})\right) \\
& \pm \frac{m_{0}}{\phi_{0}}\left[X_{A B}^{*}(\vec{p}) Y_{A B}(\vec{p})-X_{A B}(\vec{p}) Y_{A B}^{*}(\vec{p})+\left|X_{A B}(\vec{p})+Y_{A B}(\vec{p})\right|^{2}\right]^{1 / 2}
\end{aligned}\right.
$$

The mean-field sublattice magnetization $m_{A 0}=m_{B 0}=m_{0}$ and auxiliary boson field $\phi_{A 0}=$ $\phi_{B 0}=\phi_{0}$ are defined by Eqs. (15) and (14), respectively.

The product over bosonic Matsubara frequencies may be found through the Gamma function [29]

$$
\prod_{\Omega} Q(\vec{p}, \Omega)=\frac{1}{2} \prod_{\lambda=1,2} \frac{\sinh \left(\frac{\beta}{2} E_{\lambda}(\vec{p})\right)}{\sinh \left(\frac{\beta\left|\phi_{0}\right|}{2}\right)} .
$$

Then the fluctuation contribution to the free energy in the one-loop approximation is given as follows

$$
\delta F=\frac{1}{2 \beta} \sum_{\substack{\alpha=1,2 \\ \vec{p} \in R B Z}} \ln \frac{\sinh \left(\frac{\beta}{2} E_{\lambda}(\vec{p})\right)}{\sinh \left(\frac{\beta\left|\phi_{0}\right|}{2}\right)}+\frac{1}{2 \beta} \sum_{\vec{p} \in R B Z} \ln A_{0}(\vec{p}),
$$

where

$$
\begin{aligned}
A_{0}(\vec{p})=1 & +\frac{4 k_{T}^{2}(0) k_{z}^{2}\left(X_{A B}^{2}(\vec{p})+W_{A B}^{2}(\vec{p})\right)\left(X_{A B}^{* 2}(\vec{p})+W_{A B}^{* 2}(\vec{p})\right)}{\left(1-4 k_{T}^{2}(0)\right)\left|X_{A B}(\vec{p})\right|^{2}} \\
& -\frac{\left|X_{A B}(\vec{p})\right|^{2} k_{z}^{2}-4\left|W_{A B}(\vec{p})\right|^{2} k_{T}(0) k_{z}}{\left(1-4 k_{T}^{2}(0)\right)\left|X_{A B}(\vec{p})\right|^{2}} .
\end{aligned}
$$

Derivation of explicit expressions for the fluctuation contribution to the magnetization, internal energy, specific heat may be found from the free energy (35) in standard way. Note that 
the above result is derived for case of nearest-neighbor interaction, even when the magnetic order may be canted or spiral. The calculations for the case beyond the nearest-neighbor coupling are similar if the spins at the same sublattice are parallel, because the off-diagonal elements of the $3 \times 3$ matrix $\hat{D}_{\alpha \alpha}(\vec{p}, \Omega)$ vanish and the formula (22) still holds.

\section{HEISENBERG MODEL ON THE HONEYCOMB LATTICE}

As an illustration we consider the Heisenberg model on the honeycomb lattice, which is of great interest in recent years. The Hamiltonian reads:

$$
H=J \sum_{\langle i j\rangle} \vec{S}_{i} \cdot \vec{S}_{j}
$$

where $i$ and $j$ run over pairs of nearest-neighbors. The coupling constant may be ferromagnetic $(J<0)$ or antiferromagnetic $(J>0)$. The lattice structure is depicted in Fig. 1.

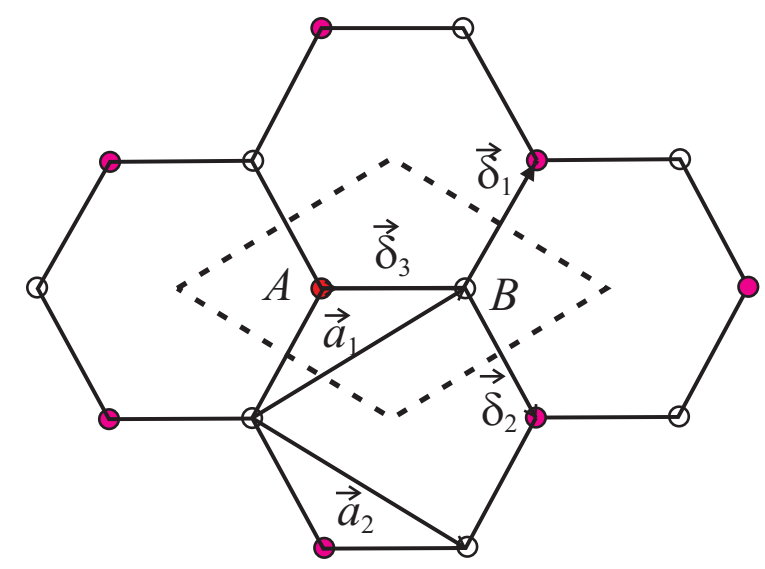

Fig. 1. The honeycomb lattice is defined by the basic vectors $\vec{a}_{1}, \vec{a}_{2}$ and two sublattices $A$ and $B$.

Setting the lattice constant $a=1$, the nearest neighbor vectors are given by:

$$
\vec{\delta}_{1}=\left(\frac{1}{2}, \frac{\sqrt{3}}{2}\right), \vec{\delta}_{2}=\left(\frac{1}{2},-\frac{\sqrt{3}}{2}\right), \vec{\delta}_{3}=(-1,0)
$$

Putting (39) into (3), after minimizing (3) with respect to $\vec{Q}$ and $\phi$ one obtains:

$$
\begin{aligned}
& \vec{Q}=(0,0), \phi=0 \text { for ferromagnetic coupling } J<0, \\
& \vec{Q}=(0,0), \phi=\pi \text { for antiferromagnetic coupling } J>0 .
\end{aligned}
$$

Paying attention to Eqs. (40) and (41), one derive from Eqs. (25):

$$
\left\{\begin{array}{l}
X_{A B}(\vec{p})=Y_{A B}(\vec{p})=-3 J \gamma(\vec{p}) \text { for } J<0 \\
X_{A B}(\vec{p})=-Y_{A B}(\vec{p})=-3 J \gamma(\vec{p}) \text { for } J>0 \\
W_{A B}(\vec{p})=0
\end{array}\right.
$$


Taking into account the above Eq. (44), from (14) and (33) we get the magnon spectrum:

$$
\begin{aligned}
& E_{1,2}(\vec{p})=3 \operatorname{Jm}_{0}\left(1 \pm|\gamma(\vec{p})|^{2}\right) \text { for } J<0 \\
& E_{1,2}(\vec{p})= \pm 3 \operatorname{Jm}_{0}\left(1-|\gamma(\vec{p})|^{2}\right) \text { for } J>0 .
\end{aligned}
$$

From Eqs. (43), (44) we can see the emergence of Dirac magnons in the honeycomb lattice. First we consider the ferromagnetic case. Expanding $\gamma(\vec{p})$ near the Dirac points

$\vec{K}_{ \pm}=\left(\frac{2 \pi}{3}, \pm \frac{2 \pi}{3 \sqrt{3}}\right)$, from (43) we obtain the linear dispersion of the so-called Dirac magnon that is similar to the spinless Dirac fermion of Bloch graphene model [24, 25]:

$$
E_{1,2}(\vec{q})=\frac{3}{2}|J| m_{0}\left(\sigma_{x} q_{x}-\tau \sigma_{y} q_{y}\right)
$$

where the $\tau= \pm 1$ correspond to the states near $\vec{K}_{ \pm}$, and $\vec{q}=\vec{p}-\vec{K}_{ \pm}$.

Next, we consider the magnon bands around the $\Gamma$ - point, $\vec{\Gamma}=(0,0)$, in the antiferromagnetic honeycomb lattice. From (44) we find the linear dispersion relation [24, 25]:

$$
E_{1,2}(\vec{q})= \pm \frac{3}{2}|J| m_{0}|\vec{q}|
$$

The results (45) and (46) are almost the same as the results obtained by applying the Holstein-Primakoff transformation of spin operators [24, 25], except the fact that in (45) and (46) the magnetization $m_{0}$ depends on temperature (15) instead of $m_{0}=s=\frac{1}{2}$. The free energy in the one loop approximation is a sum of the mean field (16) and the fluctuation contributions (36):

$$
\begin{aligned}
F= & -\frac{3 N|J| m_{0}^{2}}{2}+\frac{N}{\beta} \ln \cosh \frac{3|J| m_{0}}{2}+\frac{1}{2 \beta} \sum_{\substack{\lambda=1,2 \\
\vec{p} \in R B Z}} \ln \frac{\sinh \left(\frac{\beta}{2} E_{\lambda}(\vec{p})\right)}{\sinh \left(\frac{3 \beta|J| m_{0}}{2}\right)} \\
& +\frac{1}{2 \beta} \sum_{\vec{p} \in R B Z} \ln A_{0}(\vec{p}),
\end{aligned}
$$

where

$$
A_{0}(\vec{p})=1-\frac{9}{16} J^{2}|\gamma(\vec{p})|^{2}\left(1-4 m_{0}^{2}\right) .
$$

The magnon energy $E_{\lambda}(\vec{p})$ is defined by Eq. (43) for the ferromagnetic phase and by Eq. (44) for the antiferromagnetic phase. The first two terms are the same for both phases because it is from the mean field contribution. The magnon does not contribute to the longitudinal fluctuation, so the last term also is the same for two phases.

\section{DISCUSSIONS}

We have applied the Popov-Fedotov approach to study Heisenberg models on a non- Bravais lattice, taking into account the exac local single occupancy constraint. Parameterizing a classical ordered phase by an ordering vector and angles of spins in a unit cell and working in local coordinates we show how to derive the fluctuation contributions to the free energy for the general case of $n$ sites per unit cell. We have obtained the general analytical expressions for the nonBravais lattices with two sites in a unit cell in nearest-neighbor approximation. We have presented 
the results for the Heisenberg model on the honeycomb lattice in both cases: ferromagnetic and antiferromagnetic nearest-neighbor bondings. Taking the limit of zero temperature $T \rightarrow 0 \mathrm{~K}$ for Eq. (47) we obtain the same ground state energy derived on the linear spin wave approximation by means of the olstein-Primakoff transformation. At finite temperature the exact constraint reduces the number of states where an auxiliary fermion may thermally fluctuate into in comparizon with the case of global constraint. As a result, the free energy and, accordingly, other dynamic quantites such as the internal energy, the magnetization, the specific heat differ considerably from the corresponding quantities obtained by means of the other methods with relaxed constraint.

The results of this paper may be applied to Heisenberg models on other non-Bravais such as the Kagome, Union-Jack, checkboard, frustrated honeycomb... lattices.

\section{ACKNOWLEDGMENTS}

This research is funded by National Foundation for Science and Tecnology Development (NAFOSTED) under Grant No.103.01-2017.56.

\section{REFERENCES}

[1] A. Auerbach, Interacting electrons and quantum magnetism, Springer Verlag (1994).

[2] Yu. A. Izyumov and Y. N. Skryabin, Statistical Mechanics of Magnetically Ordered Systems Springer Verlag (1988).

[3] E. Fradkin, Field theories of condensed matter systems, Addison-Wesley Publishing Company (1991).

[4] D. P. Arovas and A. Auerbach, Phys. Rev. B 38 (1988) 316.

[5] V. N. Popov and S. A. Fedotov, Sov. Phys. JETP 67 (1988) 535.

[6] O. Veits et al., J. France 4 (1994) 493.

[7] M. N. Kicelev, Int. J. Mod. Phys. B 20 (2006) 381.

[8] S. A. Kulagin et al, Phys. Rev. Lett. 110 (2013) 070601.

[9] N. V. Prokof'ev and B. Svistunov, Phys. Rev. B 84 (2011) 073102.

[10] J. Carlstrom, J. Phys.: Condens. Matter 29 (2017) 385602.

[11] S. Tejima and A. Oguchi, J. Phys. Soc. Jpn. 64 (1995) 4923.

[12] S. Azakov et al, Int. J. Mod. Phys. B 14 (2000) 13.

[13] R. Dillenschneider and J. Richert, Eur. Phys. J. B 49 (2006) 187.

[14] Pham Thi Thanh Nga and Nguyen Toan Thang, Comm. Phys. 22 (2012) 33; Erratum, Comm. Phys. 22 (2012) 383.

[15] J. Stein and R. Oppermann, Phys. Rev. B 46 (1992) 8409.

[16] M. Bechmann and R. Oppermann, Eur. Rev. B 41 (2004) 525.

[17] M.N. Kiselev and R. Oppermann, Sov. Phys. JETP Letters 71 (2000) 250.

[18] H. T. Diep (Ed.), Frustrated Spin Systems, $2^{\text {nd }}$ ed. World Scientific, Singapore, 2013

[19] R. F. Bishop et al., Phys. Rev. B 82 (2010) 024416.

[20] R. F. Bishop et al., Phys. Rev. B 88 (2013) 214418.

[21] P. H. Li et al., J. Phys: Conf. Series 529 (2014) 012008.

[22] P. H. Li et al., Phys. Rev. B 88 (2013) 144423.

[23] S. S. Pershoguba et al., Phys. Rev. X 8 (2018) 011010.

[24] J. Fransson et al., Phys. Rev. B 94 (2016) 075401.

[25] S. A. Owerre, J. Phys.: Condens. Matter. 30 (2018) 245803.

[26] S. J. Miyake, J. Phys. Soc. Jpn. 91 (1992) 938.

[27] J. R. Silvester, Math. Gaz. 84 (2000) 460.

[28] P. D. Powell, arXiv:1112.4379 [math.RA].

[29] Pham Thi Thanh Nga and Nguyen Toan Thang, Comm. Phys. 24 (2014) 193. 